\title{
Enhanced Hybrid Compound Image Compression Algorithm Combining Block and Layer-based Segmentation
}

\author{
D. Maheswari ${ }^{1}$, Dr. V.Radha ${ }^{2}$ \\ ${ }^{1}$ Department of Computer Science, Avinashilingam Deemed University for Women , \\ Coimbatore, India \\ E-mail: mahileni@gmail.com \\ ${ }^{2}$ Department of Computer Science, Avinashilingam Deemed University for Women , \\ Coimbatore, India \\ E-mail: radharesearch@yahoo.com
}

\begin{abstract}
This paper presents an efficient compound image compression method based on both block and layerbased segmentation techniques, which introduces a new hybrid scheme for segmenting compound images. In this hybrid model the image is first segmented into five different blocks using block based classification again the overlapping block is segmented using layer based segmentation for improving the compression ratio, visual quality.
\end{abstract}

\section{KEYWORDS:}

Compound Image, Layer-based, Block-based, Segmentation, Compression.

\section{INTRODUCTION}

Compound images are different kind of images that contain both palletized regions [1], which have text or graphics, and continuous tone regions. The example of typical compound images are hybrid documents, contents captured from screen, slides, and web-pages. In this fast transmission world, compound images need to be compressed and transmitted in a secure way. Compressing compound images with a single algorithm that simultaneously meets the requirements for text, image and graphics has been elusive and thus requires new algorithms that can competently reduce the file size without degrading the quality [2]. The compound image compression performance basically depends on the segmentation result. A segmentation process is used where regions of similar data types are grouped together. After successful segmentation existing techniques that best suits each data type can be used to achieve best compression results[3].Most of the segmentation algorithms proposed in the literature belong to three categories, namely object-based, block-based and layer-based segmentation [4]. Many researchers follow either layer 
The International Journal of Multimedia \& Its Applications (IJMA) Vol.3, No.4, November 2011

or block-based methods [5][6][7] some commercial compressors like $\mathrm{DjVu}$ [8.] ,Digipaper [9] , JPEG 2000[10] also exists. This paper proposes yet another hybrid method combining both block and layer-based segmentation techniques for compressing compound image compression. The main goal is to develop a hybrid model to handle the overlapping regions of compound images. For this purpose two hybrid models were proposed. Model 1 combines block-based segmentation with wavelet packets for analyzing compression of overlapping regions. Model 2 combines both the layer -based and block -based techniques for efficient handling of overlapping images.

The paper is organized as follows: Section 1 gave a brief introduction to the topic under discussion. Section 2 discusses the compound image segmentation. Section 3 explains proposed hybrid models. Section 4 presents the results and evaluates the proposed system. Section 5 concludes the paper.

\section{SEGMENTATION TECHNIQUES IN COMPOUND IMAGE}

Compound image segmentation methods are categorized into three, researchers follows two methods.

\section{Layer- based segmentation and \\ 2. Block-based segmentation.}

This paper discusses both layer based method and block based method. A general approach used during segmentation is given in Figure 1.

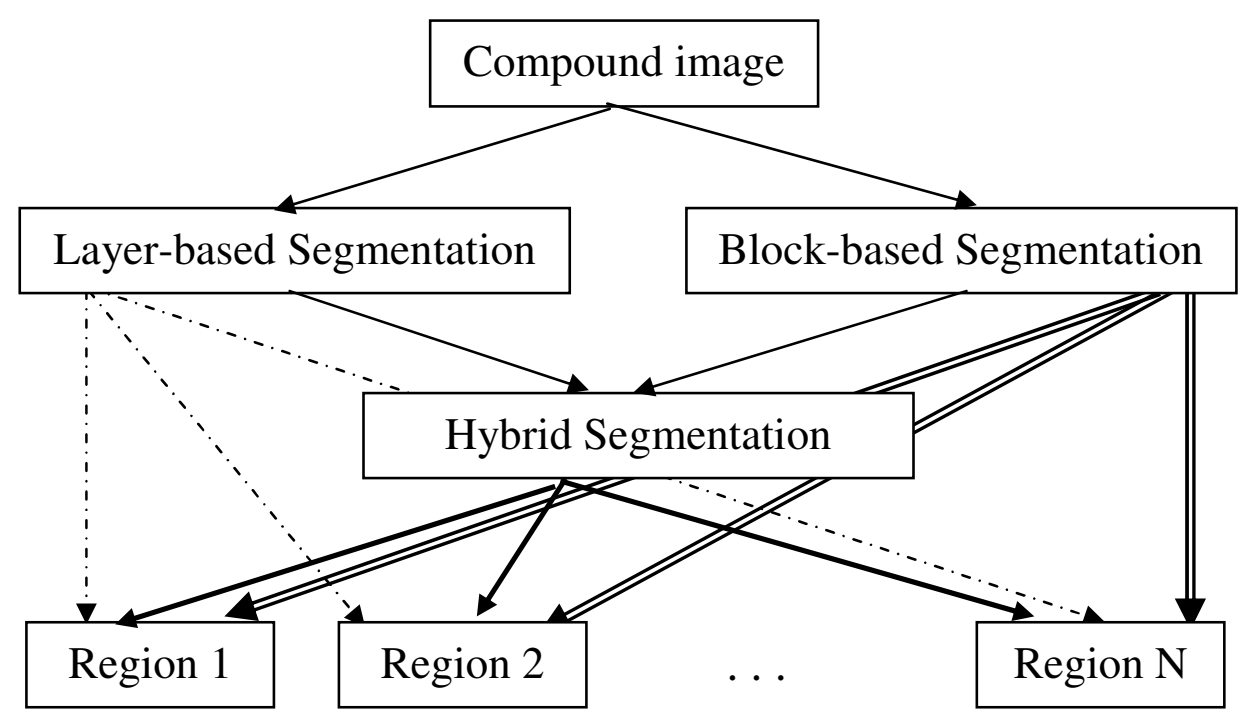

Figure 1 Segmentation Methods 
The International Journal of Multimedia \& Its Applications (IJMA) Vol.3, No.4, November 2011

\subsection{Layer-based Segmentation}

The steps followed during layer-based segmentation and compression, are,

- Segmentation of image into layers

- Production of a binary mask image

- Data filling of sparse foreground image

- Data filling of sparse background image

- Compression of each layer with an appropriate encoder

- Package of the whole representation into one meaningful stream for transmission or storage.

Most layered coding algorithms use the standard three layers Mixed Raster Content (MRC) representation.

\subsection{Block-based Segmentation}

In this technique the compound image is divided in blocks of a certain size (e.g. 8x8) and a classification method is applied to the block to segment the image into blocks, which can later be compressed using different techniques. The classification information is also coded before coding the block pixels and usually it depends only on the values of the pixels inside a block and possibly the decision on the neighboring blocks.

\section{HYBRID MODELS}

The block and layer-based methods separately gives better performance. Combining the two segmentation methods gives much better results than the individuals. For this purpose the model 1 and model 2 are proposed.

\subsection{Model 1 -Using Wavelet Packet Tree}

The Model 1 uses block classification method to identify various blocks in the compound image. As the first step, the image pixels are grouped into three classes, namely Low-Gradient, MidGradient and High-Gradient pixels. In this model the compound image is first divided into 16x16 blocks. Using the gradient values and a combination of decision rules, the block is classified into five different regions, namely, background block, text block, picture block, graphics block and overlapping blocks. Out of all the five blocks, the main challenge was faced with the overlapping block, which had mixed contents. For this purpose a wavelet packet-based compression technique is used. The overall process is given in Figure 2. 
The International Journal of Multimedia \& Its Applications (IJMA) Vol.3, No.4, November 2011

Figure 2: The Proposed Model 1 Segmentation Scheme

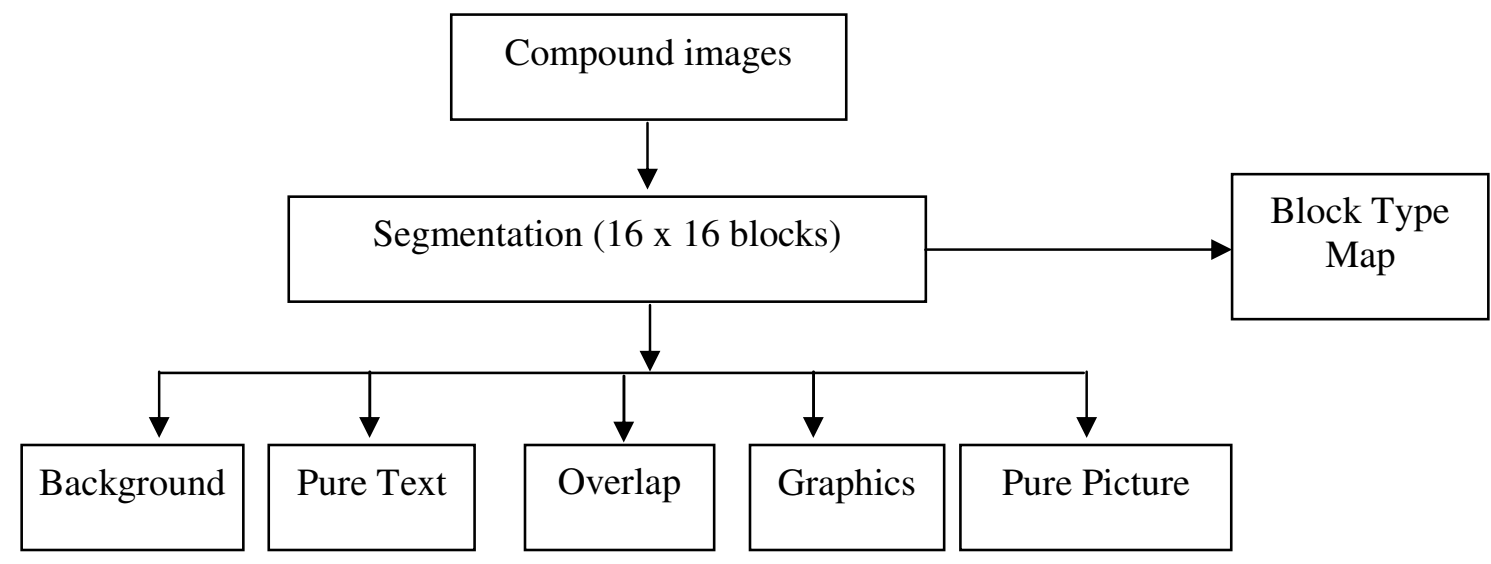

During segmentation, two features, namely, histogram and gradient of the block is used. Using the gradient values, the histogram distribution for each pixel group is computed.

For this purpose the following decision rules are used, which are shown in Figure 3.

$\begin{array}{|cl|}\text { Rule } 1 \text { : If } H G+L G<T_{1} & \rightarrow \text { Picture block } \\ \text { Rule } 2: \text { If } H G<T 2 \text { and } L G>T 3 \text { and Gray level Range } & >T 4 \\ \text { Else } & \rightarrow \text { Background block } \\ \text { Rule } 3 \text { : If Major colors Number } \leq 2 \text { and } H G P>T 6 & \rightarrow \text { Picture block } \\ \text { Else if colors } \geq 2 & \rightarrow \text { Graphics block } \\ \text { Else } & \rightarrow \text { Picture bock } \\ \text { Rule } 4: \text { If } H G \geq T 7 & \rightarrow \text { Overlap block } \\ \text { Else } & \rightarrow \text { Picture block } \\ \text { Figure 3: Decision Rules of Model } 1 \\ \end{array}$

The overlapping block, which cannot be compactly represented both in spatial and frequency domain contain strong high frequency signals due to text edges. In order to fully utilise these during compression a wavelet packet -based compression is used. Orthonormal wavelet packets are created by decomposing a signal into background (S) and detail (D) subbands corresponding to different orthogonal, critically sampled frequency bands. 
The International Journal of Multimedia \& Its Applications (IJMA) Vol.3, No.4, November 2011

\subsubsection{Wavelet Packet and Wavelet Packet Tree}

The wavelet packet is as same as wavelet; the only difference is that wavelet packet offers a more complex and flexible analysis because in wavelet packet analysis the details as well as the approximation are split.

The wavelet packet tree for 3-level decomposition is shown in Figure 4.

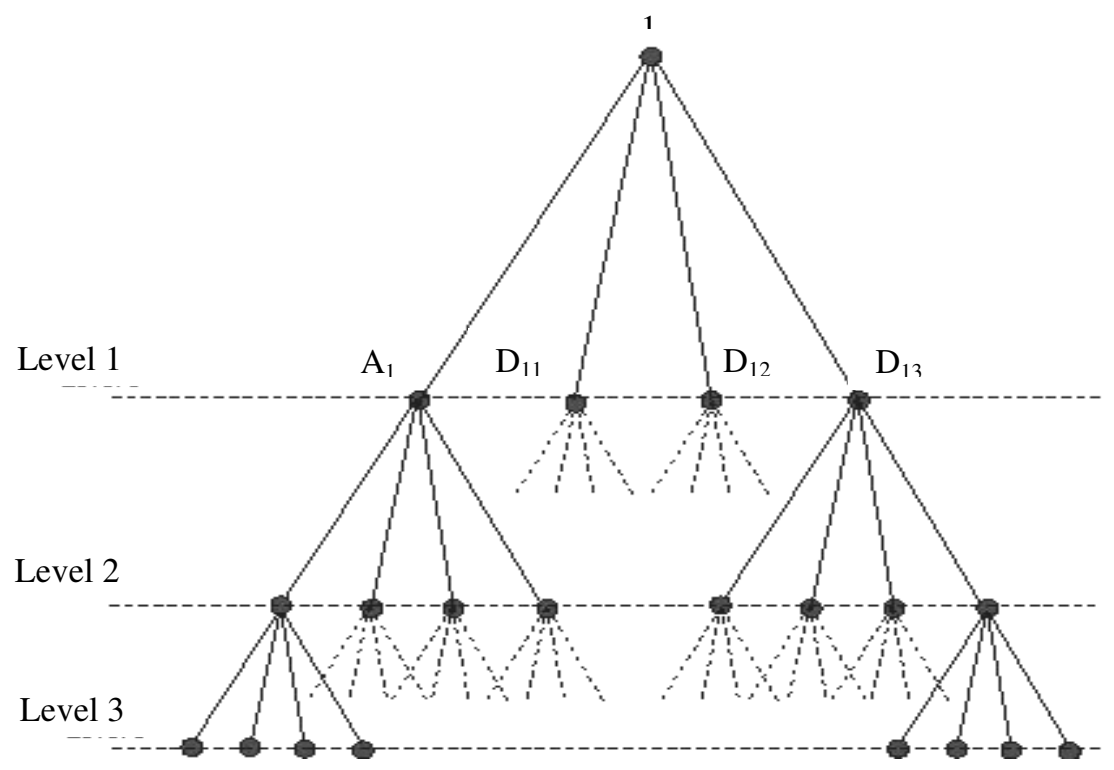

Figure 4 : Wavelet Packet Tree Decomposition

The entropy function used is the simple threshold entropy, which is used to construct the best tree. After best tree construction, a cost thresholding function (Equation 1) is used to compress the data.

$$
\text { Entropy }=\sum_{i=0}^{N-1} a b s\left(X_{i}\right)>T
$$

where $X_{i}$ is the $i^{\text {th }}$ coefficient of sub-band, $\mathrm{N}$ is the length of sub-band and $\mathrm{T}$ is the user-defined threshold.

The tree construction procedure is as follows: Information content of decomposed component (approximation and details) may be greater than the information content of components, which has been decomposed. This method reduces the time complexity of wavelet packets decomposition and selects the sub-bands that have vital information. The decision of further decomposition and cost calculation is based on a strategy, which decides during run time whether to retain or prune the decomposed components. After the best basis has been selected based on cost function, the image is represented by a set of wavelet packets coefficients, which is further 
The International Journal of Multimedia \& Its Applications (IJMA) Vol.3, No.4, November 2011

compressed using run length encoder to give better overall compression. The algorithm is given in Figure 5.

Let level counter $=1$

1. Let the current node = input image.

2. Decompose current node using wavelet packet tree.

3. Find the entropy of current node.

4. Find the entropy of decomposed components.

5. Compare the entropy of parent node with the sum of the entropy of child node. If the sum of the entropy of child nodes is less than that of parent node, then child node will be considered as a leaf node of a tree and repeat the steps 3-6 for each child nodes considering it as current node. Otherwise parent node acts as a leaf node of a tree.

6. Stop.

Figure 5: Algorithm for Overlapping Block

\subsection{Model 2- Combination of layer based and Block-based Method}

In the model 2, the two techniques layer-based and block-based are combined to form a hybrid compound image compression technique. The method proposed uses model 1 for segmentation, but differs in the handling of the overlapping block. The overlapping block is dealt with separately and uses the layer-based approach to segment the block into three layers text, picture and mask. Then the various compression schemes suiting the need of block or layer are applied. This model combines the advantages of both layer-based and block-based approaches and hence can be considered as an improvement of both the approaches.

The segmentation scheme for hybrid model is given in Figure 6.

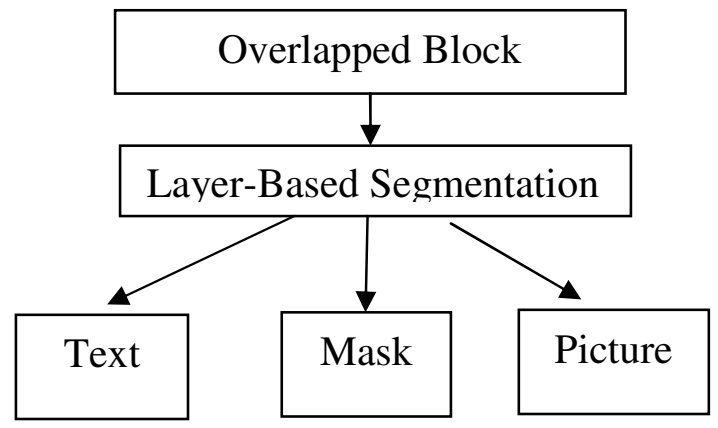

Figure 6: Hybrid Model Segmentation Scheme 
The International Journal of Multimedia \& Its Applications (IJMA) Vol.3, No.4, November 2011

\subsubsection{Layer-Based Segmentation}

Most layered coding algorithms use the standard three layers Mixed Raster Content (MRC) representation. In this segmentation the image is segmented into text, mask and graphics layer. Each layer is compressed using different compressors. The mask layer contains the contours of text and other fine image structures.

JBIG (Joint Bi-level Image Experts Group) algorithm is used to lossless compress the mask layer .The Text layer is compressed using token based coder. Mask layer is compressed using JBIG coder and the graphics layer is compressed using the JPEG coder. The following Figure 7 shows the process of proposed layer-based segmentation method.

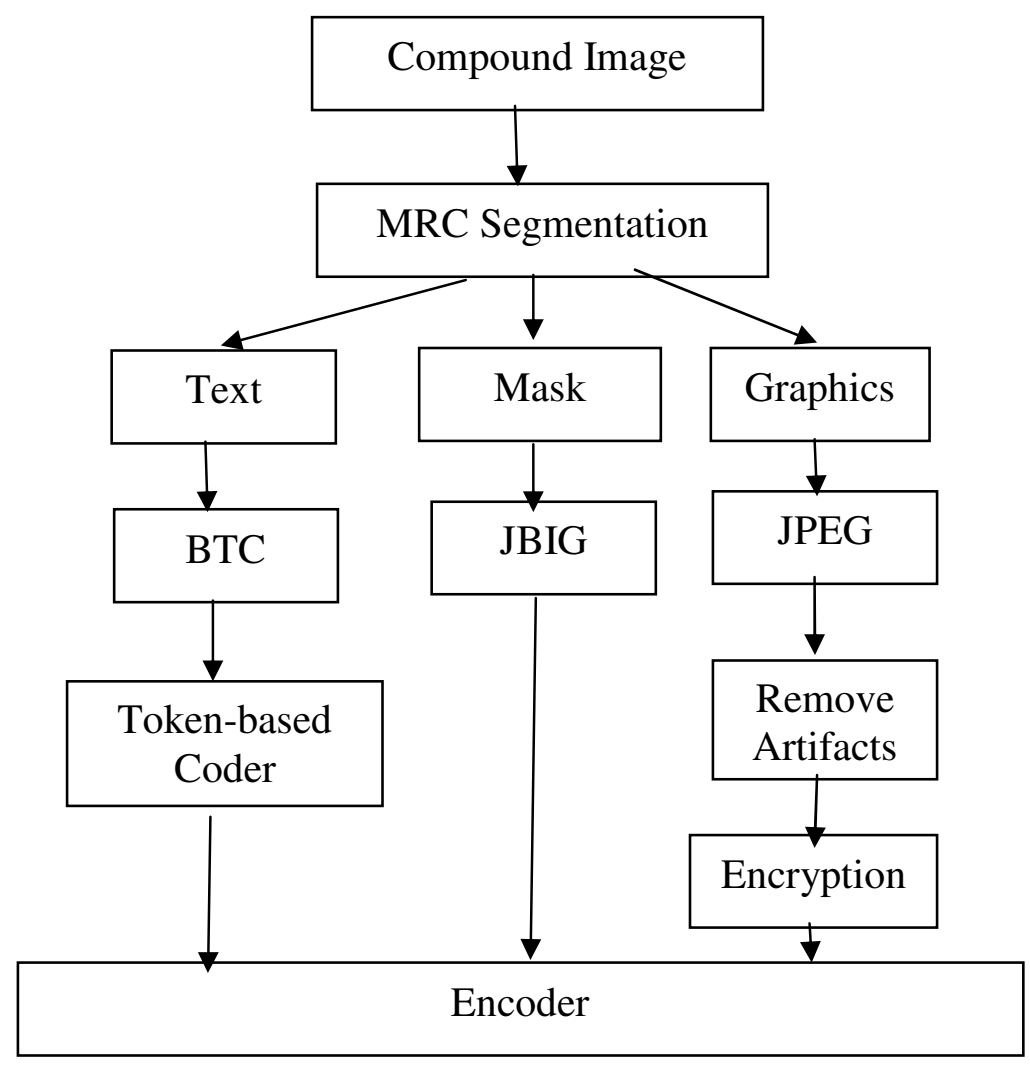

Figure 7: Layer-based Segmentation

\section{ENCRYPTION}

Encryption is the process of transforming the information to ensure security during transmission and storage. Encryption converts an image to another form which is very different from the original image and which will be hard to understand. Decryption, on the other hand, performs inverse of encryption to retrieve the original image from the encrypted one. There are various image encryption systems to encrypt and decrypt data and there is no single encryption algorithm that satisfies all types of images. In this paper the encryption is to be applied to segmented region rather than the whole image. For protecting text region, a Block Transformation Coding (BTC) is 
The International Journal of Multimedia \& Its Applications (IJMA) Vol.3, No.4, November 2011

used. The picture/image and graphics region of the compound image is protected using chaos theory and sorting transformation algorithm.

\section{EXPERIMENTAL RESULTS}

The main objective of the tests was to find which of the proposed algorithms is best suited for compound color image compression.

\subsection{Test Images}

In order to verify the performance of the proposed schemes, experimental evaluation is performed using a series of different benchmark images. The experimentation is done in three types of compound images, which are Scanned document images, Computer generated images and Photographic images with compound nature. The following figure 8 shows the test images.

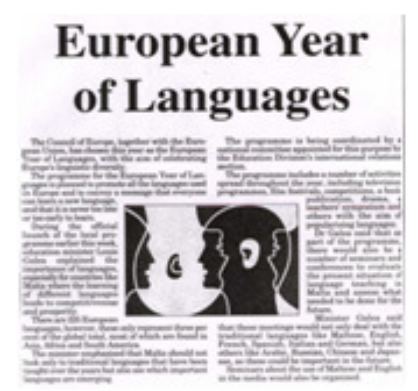

(a)

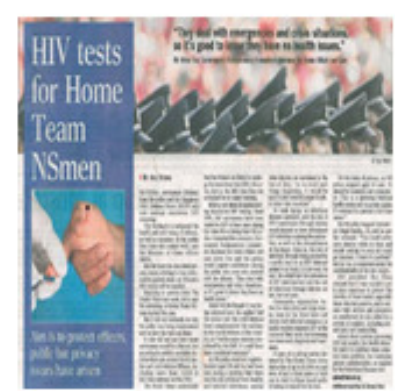

(b)

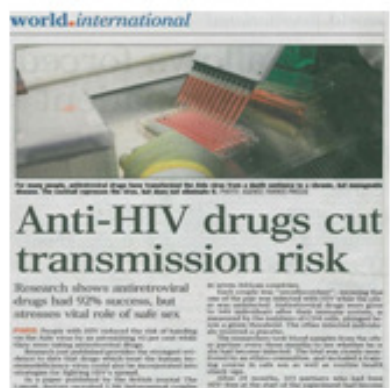

(c)

\section{Document and scanned Test Images}

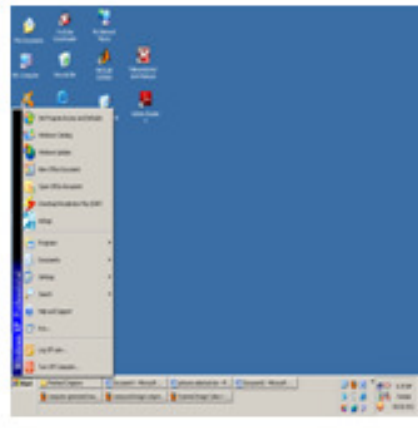

(d)

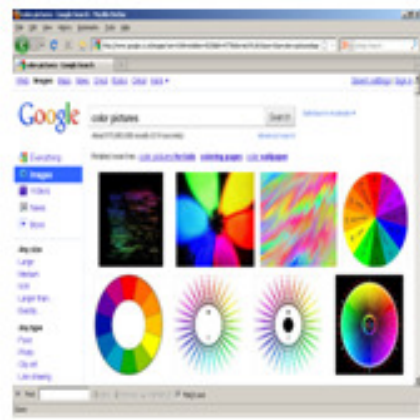

(e)

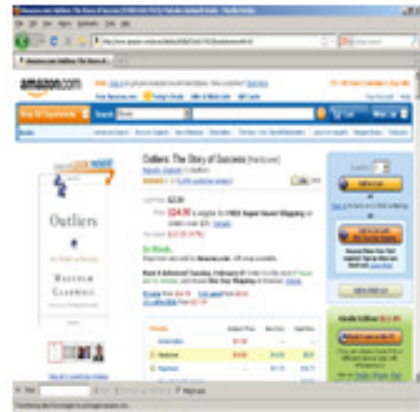

(f)

Computer Generated Images 


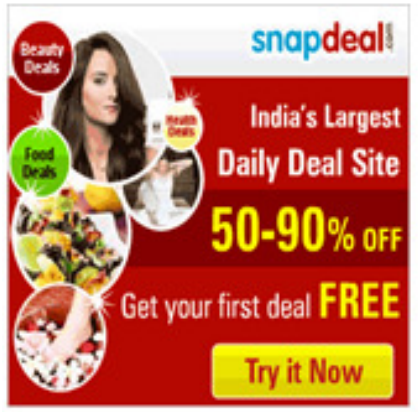

(g)

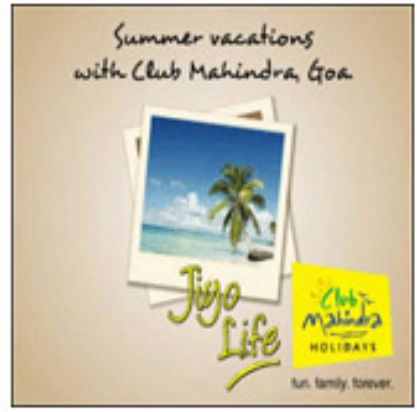

(h)

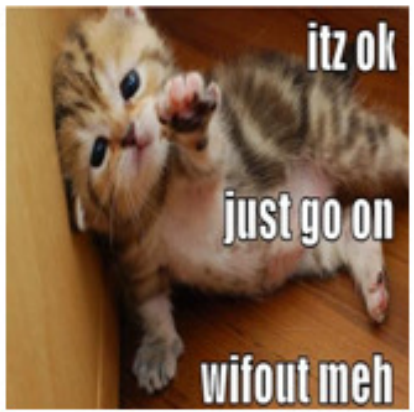

(i)

\section{Photographic Compound Natured Images}

Figure 8: Test Images

For experimentation, 9 images were selected and the selection was done in such a way that they were combination of all classes of compound images. All images were of the size $256 \times 256$ pixels.

\subsection{Performance Metrics}

The performance of the proposed models was evaluated using different parameters like compression ratio, compression and decompression time. One of the objectives of the proposed compression models is to maintain the visual quality of the decompressed image. The quality of the decompressed image was ascertained by using the quality metric Peak Signal to Noise Ratio (PSNR).

\section{Compression Ratio}

The compression ratio of the test images were presented in Table I. The proposed models are compared with traditional compressors JPEG and $\mathrm{DjVu}$.

Table I Compression Ratio(\%)

\begin{tabular}{|c|l|l|l|l|l|}
\hline Images & $\begin{array}{l}\text { Layer- } \\
\text { based }\end{array}$ & Model 1 & Model 2 & JPEG & $\mathrm{DjVu}$ \\
\hline $\mathrm{a}$ & 45.79 & 45.02 & 47.91 & 37.43 & 39.89 \\
\hline $\mathrm{b}$ & 40.36 & 39.56 & 43.02 & 35.00 & 36.51 \\
\hline $\mathrm{c}$ & 39.91 & 39.12 & 42.51 & 34.78 & 36.24 \\
\hline $\mathrm{d}$ & 38.99 & 42.77 & 45.91 & 36.39 & 37.67 \\
\hline $\mathrm{e}$ & 38.16 & 39.12 & 40.32 & 36.47 & 37.12 \\
\hline $\mathrm{f}$ & 37.14 & 39.25 & 41.67 & 35.92 & 36.23 \\
\hline $\mathrm{g}$ & 40.68 & 42.26 & 44.32 & 35.64 & 37.62 \\
\hline $\mathrm{h}$ & 42.37 & 43.38 & 45.66 & 36.60 & 38.84 \\
\hline $\mathrm{i}$ & 39.59 & 40.67 & 43.95 & 36.67 & 38.74 \\
\hline
\end{tabular}


The International Journal of Multimedia \& Its Applications (IJMA) Vol.3, No.4, November 2011

From the above Table I, it can be seen that the proposed Model 2 produces good compression ratio for all the test images. However the proposed models Model 1 and layer-based gives better results followed by Model 2.

\section{Compression Time and Decompression Time}

The following Table II shows the result of compression and decompression time for all the models. The compression and decompression time are the time taken for encoding and decoding an image.

Table II Compression and Decompression Time (Seconds)

\begin{tabular}{|c|l|l|l|l|l|l|l|l|l|l|}
\hline $\begin{array}{c}\text { Image } \\
\text { s }\end{array}$ & \multicolumn{2}{|c|}{ Layer-based } & \multicolumn{2}{c|}{ Model 1 } & \multicolumn{2}{c|}{ Model 2 } & \multicolumn{2}{c|}{ JPEG0. } & \multicolumn{2}{c|}{ DjVu } \\
\hline & CT & DT & CT & DT & CT & DT & CT & DT & CT & DT \\
\hline a & 0.68 & 0.53 & 0.77 & 0.65 & 0.75 & 0.62 & 1.02 & 0.79 & 0.89 & 0.73 \\
\hline b & 0.69 & 0.58 & 0.72 & 0.66 & 0.72 & 0.64 & 1.02 & 0.82 & 0.89 & 0.75 \\
\hline c & 0.65 & 0.58 & 0.71 & 0.65 & 0.71 & 0.63 & 0.98 & 0.83 & 0.84 & 0.77 \\
\hline d & 0.65 & 0.59 & 0.73 & 0.67 & 0.72 & 0.66 & 0.87 & 0.83 & 0.83 & 0.72 \\
\hline e & 0.77 & 0.66 & 0.82 & 0.74 & 0.81 & 0.72 & 0.89 & 0.83 & 0.85 & 0.75 \\
\hline f & 0.75 & 0.65 & 0.84 & 0.75 & 0.83 & 0.73 & 0.96 & 0.89 & 0.93 & 0.75 \\
\hline g & 0.67 & 0.57 & 0.72 & 0.65 & 0.71 & 0.62 & 0.92 & 0.84 & 0.82 & 0.76 \\
\hline h & 0.63 & 0.56 & 0.73 & 0.64 & 0.71 & 0.63 & 0.93 & 0.84 & 0.84 & 0.76 \\
\hline i & 0.65 & 0.57 & 0.73 & 0.63 & 0.72 & 0.62 & 0.94 & 0.75 & 0.89 & 0.71 \\
\hline
\end{tabular}

\section{Peak Signal to Noise Ratio}

The PSNR is used to judge the quality of decompressed image with that of the original image. The Table III compares the PSNR values of proposed algorithms.

TABLE III Peak Signal to Noise Ratio

\begin{tabular}{|c|c|c|c|c|c|}
\hline Images & $\begin{array}{c}\text { Layer- } \\
\text { based }\end{array}$ & Model 1 & Model 2 & JPEG & $\mathrm{DjVu}$ \\
\hline $\mathrm{a}$ & 44.86 & 43.67 & 46.69 & 34.86 & 38.68 \\
\hline $\mathrm{b}$ & 42.86 & 41.17 & 44.97 & 34.42 & 36.23 \\
\hline $\mathrm{c}$ & 41.79 & 40.83 & 43.62 & 33.21 & 35.23 \\
\hline $\mathrm{d}$ & 38.88 & 44.97 & 46.14 & 35.08 & 36.68 \\
\hline $\mathrm{e}$ & 36.54 & 40.12 & 41.99 & 33.55 & 35.34 \\
\hline $\mathrm{f}$ & 37.12 & 40.62 & 41.65 & 31.87 & 33.55 \\
\hline $\mathrm{g}$ & 41.01 & 43.32 & 44.60 & 33.67 & 35.96 \\
\hline $\mathrm{h}$ & 41.98 & 43.95 & 44.68 & 33.23 & 35.14 \\
\hline $\mathrm{i}$ & 41.83 & 43.59 & 94.23 & 34.48 & 36.13 \\
\hline
\end{tabular}

The high PSNR value is obtained by the proposed model 2 compared with other models. 
The International Journal of Multimedia \& Its Applications (IJMA) Vol.3, No.4, November 2011

To show the improvement of the proposed algorithms, a Visual comparison of the three images $(\mathrm{c}, \mathrm{e}, \mathrm{h})$, before and after compression is shown in figure 9.
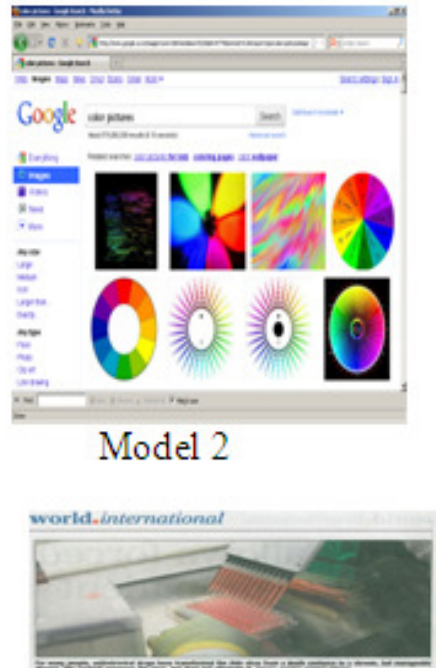

Anti-HIV drugs cut transmission risk

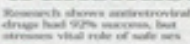

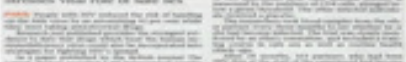

Model 2

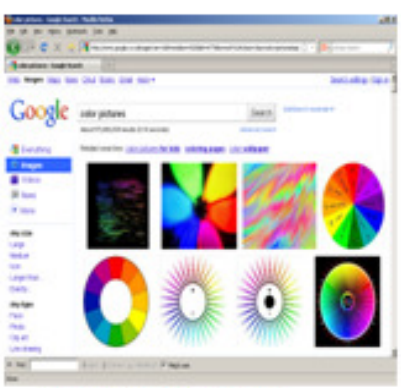

Original Image

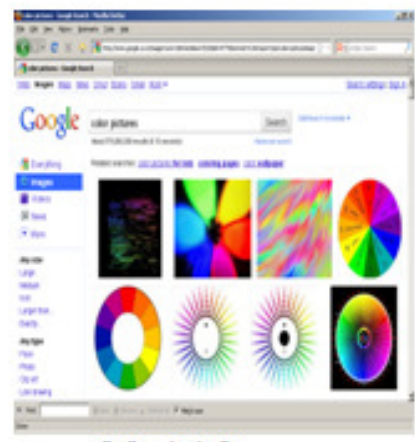

Model 2

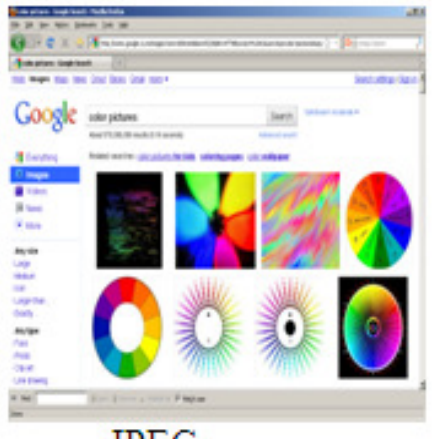

JPEG
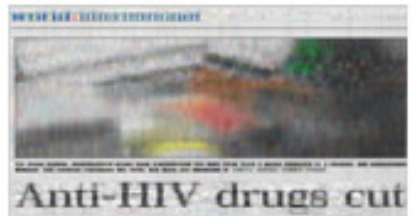
transmission risik

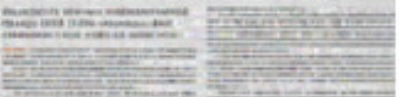

JPEG

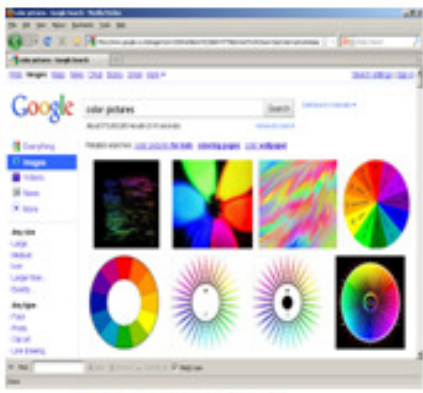

Laver-based
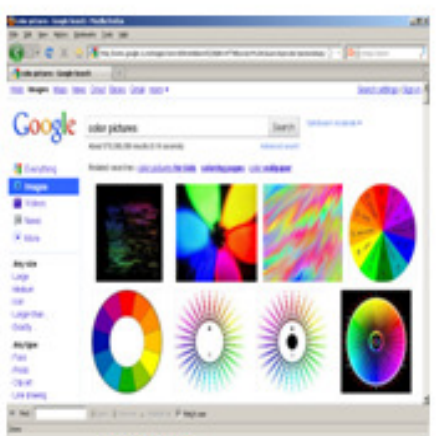

JPEG

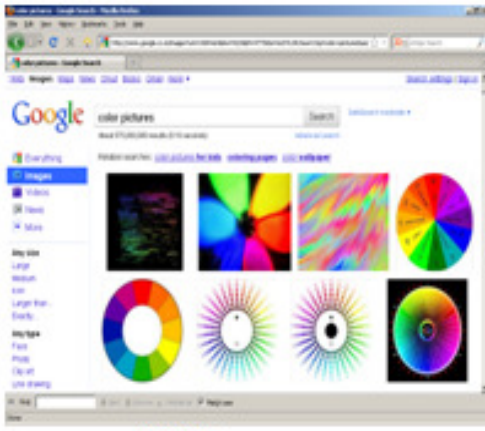

$\mathrm{Dj} \mathrm{Vu}$

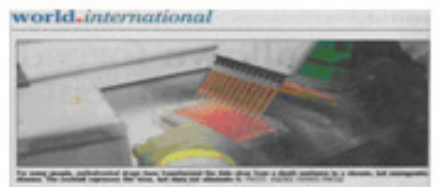

Anti-HIV drugs cut transmission risk

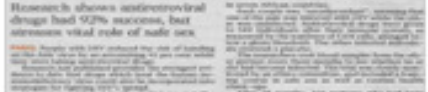

$\mathrm{DjVu}$

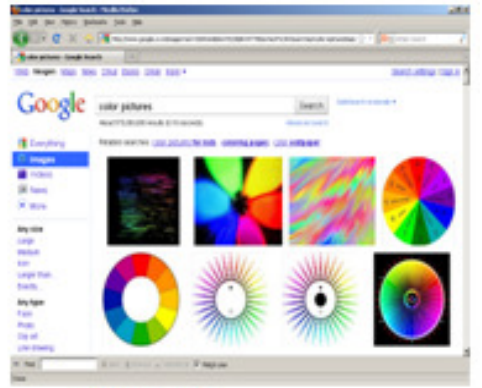

Model 1

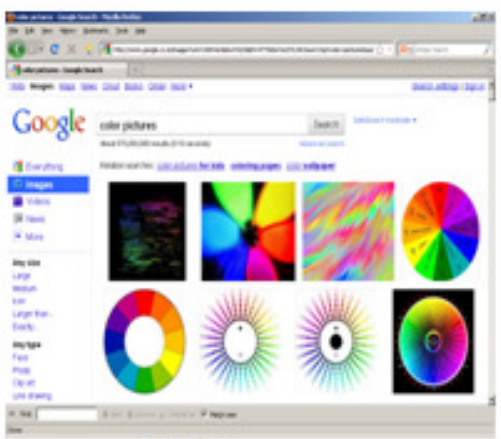

$\mathrm{DjVu}$

Figure 9: Visual Comparison of Test Images 
From the above results, Model 2 achieves better results followed by Model 1 when compared with other models.

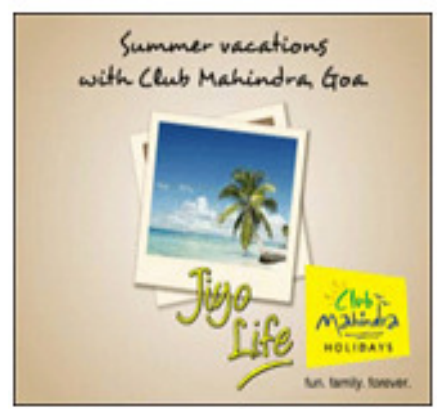

Original Image

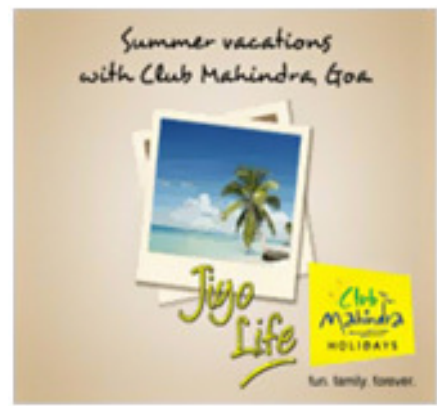

Model 2

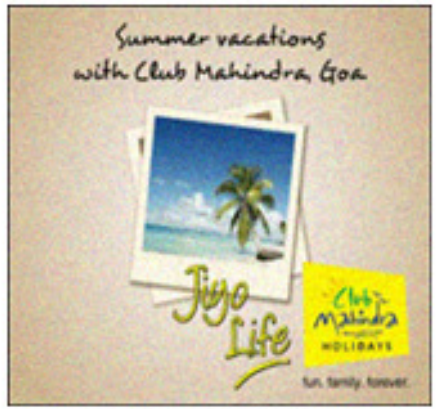

Layer-based

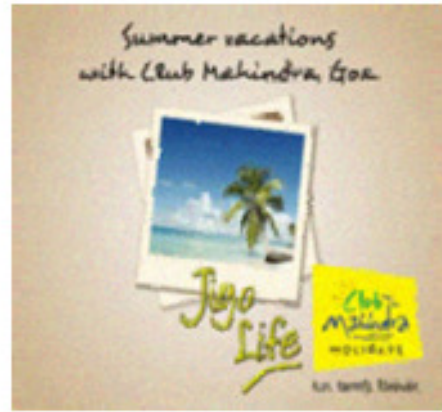

JPEG

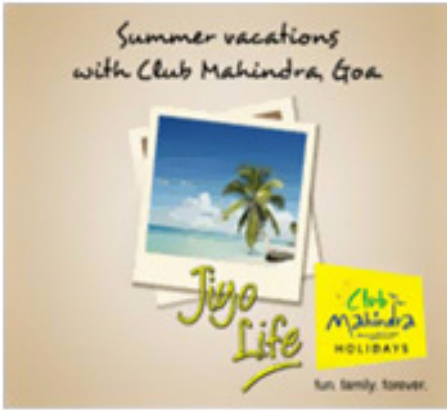

Model 1

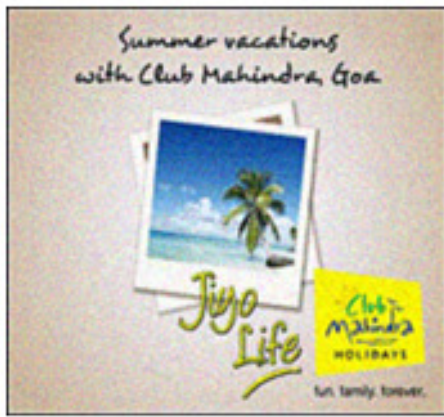

$\mathrm{DjVul}$

\section{CONCLUSION}

This paper proposed two hybrid compound image compression models. In this The first model divides the image into five blocks and applies block-based classification.The second model uses block-based segmentation which is used in the first model and for overlapping block it again applies layer-based segmentation. The proposed models are compared with traditional JPEG and $\mathrm{Dj} \mathrm{Vu}$. During experimentation, it was found that the proposed Model 2 produces good results compared with other models.

\section{REFERENCES}

[1] Dong Liu, Wenpeng Ding, Yuwen He, Feng Wu (2006) Quality-biased Rate Allocation for Compound Image Coding with Block Classification, 0-7803-9390-2/06/\$20.00 @2006 IEEE,Pp 49474950 .

[2] Maheswari, D. and Radha V. (2010) Enhanced Layer Based Compound Image Compression, Proceedings of the First Amrita ACM-W celebration of Women in Computing ISBN:978-1-4503 0194-7, Pp. 209-216. 
The International Journal of Multimedia \& Its Applications (IJMA) Vol.3, No.4, November 2011

[3] Maheswari, D. and Radha V. (2010) Secure Layer Based Compound Image Compression using XML Compression, 2010 IEEE International Conference on Computational Intelligence and Computing Research, ISBN:978-1-4244-5966-7,Pp.494-498.

[4] Ding, W., Lu, Y. and Wu, F. 2007. Enable efficient compound image compression in H.264/AVC intra coding, IEEE International Conference on Image Processing, Vol. 2, pp. II - 337 - II - 340.

[5] Radha, V., Aparna R., and Maheswari, D. 2010.Performance evaluation of H.264/AVC intra compound image compression system, International Journal of Computer Applications, Foundation of Computer Science, Vol. 1, No.10, pp. 48-54.

[6] Xi, Q., Wu, X. and Zhang, S. 2009. Compound Image Compression with Multi-step Text Extraction Method, Fifth International Conference on Intelligent Information Hiding and Multimedia Signal Processing, pp.1270-1273.

[7] Zaghetto, A. and de Queiroz, R.L. 2008. Iterative pre-and post-processing for MRC layers of scanned documents, 15th IEEE International Conference on Image processing, ICIP 2008, pp. 1009-1012.

[8] Bottou, L., Haffner, P., Howard, P., Simard, P., Bengio, Y. and LeCun, Y. 1998. High quality document image compression using DjVu, Journal of Electronic Imaging, Vol. 7, No.3, pp. 410-425.

[9] Huttenlocher, D., Felzenszwalb, P. and Rucklidge, W.1999. DigiPaper: A versatile color document image representation, Proc. ICIP, Vol. I, pp. 219-223.

[10] Sharpe, L.H. and Buckley, R. 2000. JPEG 2000 .jpm file format: a layered imaging architecture for document imaging and basic animation on the web, Proceedings of SPIE, Vol.4115, pp. 464-475.

[11] Reddy, P.V., Sharma, K.V., Mallesham, P. and Radhadevi, P. (2010) Secure Image Transmission Through Unreliable Channels, International Journal on Computer Science and Engineering, Vol. 02, No. 06, Pp. 2053-2058. 